PROCEEDINGS OF THE

AMERICAN MATHEMATICAL SOCIETY

Volume 139, Number 4, April 2011, Pages 1333-1345

S 0002-9939(2010)10787-6

Article electronically published on November 30, 2010

\title{
NEUMANN PROBLEM ON A HALF-SPACE
}

\author{
FUMIYAMA SHU, MASAKI TANAKA, AND MINORU YANAGISHITA \\ (Communicated by Mario Bonk)
}

\begin{abstract}
In this paper, a solution of the Neumann problem on a half-space for a slowly growing continuous boundary function is constructed by the generalized Neumann integral with this boundary function. The relation between this particular solution and certain general solutions is discussed. A solution of the Neumann problem for any continuous boundary function is also given explicitly by the Neumann integral with the generalized Neumann kernel depending on this boundary function.
\end{abstract}

\section{INTRODUCTION}

Let $n$ be a positive integer satisfying $n \geq 2$. Let $\mathbf{R}^{n+1}$ be the $(n+1)$-dimensional Euclidean space. A point in $\mathbf{R}^{n+1}$ is represented by

$$
M=(X, y)=\left(x_{1}, \ldots, x_{n}, y\right)
$$

with

$$
|M|=\left(x_{1}^{2}+\ldots+x_{n}^{2}+y^{2}\right)^{\frac{1}{2}} .
$$

By $\partial E$ we denote the boundary of a subset $E$ of $\mathbf{R}^{n+1}$. The sphere of radius $r$ centered at the origin of $\mathbf{R}^{n+1}$ is represented by $S_{n+1}(r)$. By $\mathbf{T}_{n+1}$ we denote the open half-space

$$
\left\{M=(X, y) \in \mathbf{R}^{n+1}: y>0\right\} .
$$

Then $\partial \mathbf{T}_{n+1}$ is identified with $\mathbf{R}^{n}$ and the $n$-dimensional Lebesgue measure at $N \in \partial \mathbf{T}_{n+1}$ is denoted by $d N$. When $g$ is a function defined on

$$
\sigma_{n+1}(r)=\mathbf{T}_{n+1} \cap S_{n+1}(r) \quad(r>0),
$$

we define the mean of $g$ as follows:

$$
\mathcal{M}(g ; r)=2\left(s_{n+1} r^{n}\right)^{-1} \int_{\sigma_{n+1}(r)} g(M) d \sigma_{M} \quad(r>0),
$$

where $s_{n+1}$ is the surface area of $S_{n+1}(1)\left(\right.$ the $(n+1)$-dimensional unit sphere $\left.\mathbf{S}^{n}\right)$ and $d \sigma_{M}$ is the surface element on $S_{n+1}(r)$ at $M \in \sigma_{n+1}(r)$.

Let $f$ be a continuous function defined on $\partial \mathbf{T}_{n+1}$. A solution of the Neumann problem on $\mathbf{T}_{n+1}$ for $f$ is a harmonic function $h$ in $\mathbf{T}_{n+1}$ such that

$$
\lim _{M \in \mathbf{T}_{n+1}, M \rightarrow N} \frac{\partial}{\partial y} h(M)=f(N)
$$

Received by the editors November 20, 2009.

2000 Mathematics Subject Classification. Primary 31B05; Secondary 31B20.

Key words and phrases. Half-space, Neumann problem, Neumann integral.

(C)2010 American Mathematical Society Reverts to public domain 28 years from publication 
for every point $N \in \partial \mathbf{T}_{n+1}$. Armitage proved

Theorem A (Armitage [1, Theorem 1 and Remarks). Let $f$ be a continuous function on $\partial \mathbf{T}_{n+1}=\mathbf{R}^{n}$ such that

$$
\int_{\mathbf{R}^{n}}(1+|N|)^{1-n}|f(N)| d N<\infty .
$$

Then a solution of the Neumann problem on $\mathbf{T}_{n+1}$ for $f$ is given by the Neumann integral $I_{f}$ for $f$,

$$
I_{f}(M)=-\alpha_{n+1} \int_{\mathbf{R}^{n}}|M-N|^{1-n} f(N) d N \quad\left(M \in \mathbf{T}_{n+1}\right)
$$

which satisfies

$$
\mathcal{M}\left(\left|I_{f}\right| ; r\right)=O(1) \quad(r \rightarrow \infty)
$$

where $\alpha_{n+1}=2\left\{(n-1) s_{n+1}\right\}^{-1}$.

The following result deals with a type of uniqueness of solutions for the Neumann problem on $\mathbf{T}_{n+1}$.

Theorem B (Armitage [1, Theorem 3). Let $k$ be a positive integer and $f$ be a continuous function on $\partial \mathbf{T}_{n+1}$ satisfying (1.1). If $h$ is a solution of the Neumann problem on $\mathbf{T}_{n+1}$ for $f$ satisfying

$$
\mathcal{M}\left(h^{+} ; r\right)=o\left(r^{k}\right) \quad(r \rightarrow \infty)
$$

then $h$ is given by

$$
h(M)=I_{f}(M)+ \begin{cases}C & (k=1), \\ \Pi(X)+\sum_{j=1}^{\left[\frac{k}{2}\right]} \frac{(-1)^{j}}{(2 j) !} y^{2 j} \Delta^{j} \Pi(X) & (k \geq 2)\end{cases}
$$

for any $M=(X, y) \in \mathbf{T}_{n+1}$, where $h^{+}$is the positive part of $h$,

$$
\Delta^{j}=\left(\frac{\partial^{2}}{\partial x_{1}^{2}}+\frac{\partial^{2}}{\partial x_{2}^{2}}+\cdots+\frac{\partial^{2}}{\partial x_{n}^{2}}\right)^{j} \quad(j=1,2, \ldots),
$$

$C$ is a constant and $\Pi$ is a polynomial of $X=\left(x_{1}, x_{2}, \ldots, x_{n}\right) \in \mathbf{R}^{n}$ of degree less than $k$ in $\partial \mathbf{T}_{n+1}$.

Gardiner [7, Theorem 1] gave a solution of the Neumann problem for any continuous function on $\partial \mathbf{T}_{n+1}$. His solution is constructed using approximation of functions, and hence it is not explicit. In this paper, we will explicitly give a solution of the Neumann problem for any continuous function on $\partial \mathbf{T}_{n+1}$ in the same way as Finkelstein and Scheinberg [6] and Yoshida [10] did in the case of the Dirichlet Problem. To do this, Theorem A will be extended by defining generalized Neumann integrals for continuous functions under less restricted conditions than (1.1) (Theorem 1). Siegel and Talvila [9] defined a more complicated generalized Neumann integral for their purpose. But our generalized Neumann integral is much simpler than theirs. By using Theorem 1, we shall give a solution of the Neumann problem for any continuous function on $\partial \mathbf{T}_{n+1}$. Our solution is much simpler than the solution given by Gardiner (Theorem 2). We shall also extend Theorem B (Theorem 3). 


\section{Statements of Results}

Let $M$ and $N$ be two points in $\mathbf{T}_{n+1}$ and $\partial \mathbf{T}_{n+1}$, respectively. By $\langle M, N\rangle$ we denote the usual inner product in $\mathbf{R}^{n+1}$. We note that

$$
|M-N|^{1-n}=\sum_{k=0}^{\infty} c_{k, n+1}|N|^{1-k-n}|M|^{k} L_{k, n+1}(\rho) \quad(|M|<|N|),
$$

where

$$
\rho=\frac{\langle M, N\rangle}{|M||N|}, \quad c_{k, n+1}=\left(\begin{array}{c}
k+n-2 \\
k
\end{array}\right)
$$

and $L_{k, n+1}$ is the $(n+1)$-dimensional Legendre polynomial of degree $k$. We remark that $L_{k, n+1}(1)=1, L_{k, n+1}(-1)=(-1)^{k}, L_{0, n+1}=1$ and $L_{k, n+1}(t)=t$ (see Armitage [3, p. 55]).

Let $l$ be a non-negative integer. We set

$$
V_{l, n+1}(M, N)= \begin{cases}-\alpha_{n+1} \sum_{k=0}^{l-1} c_{k, n+1}|N|^{1-k-n}|M|^{k} L_{k, n+1}(\rho) & (|N| \geq 1, \quad l \geq 1), \\ 0 & (|N|<1, \quad l \geq 1), \\ 0 & (l=0)\end{cases}
$$

for any $M \in \mathbf{T}_{n+1}$ and any $N \in \partial \mathbf{T}_{n+1}$. The generalized Neumann kernel $K_{l, n+1}(M, N)\left(M \in \mathbf{T}_{n+1}, N \in \partial \mathbf{T}_{n+1}\right)$ is defined by

$$
K_{l, n+1}(M, N)=K_{0, n+1}(M, N)-V_{l, n+1}(M, N) \quad(l \geq 0),
$$

where

$$
K_{0, n+1}(M, N)=-\alpha_{n+1}|M-N|^{1-n} .
$$

Since $|M|{ }^{k} L_{k, n+1}(\rho)(k \geq 0)$ is harmonic in $\mathbf{T}_{n+1}$ (Armitage [3, Theorem D]), $K_{l, n+1}(\cdot, N)$ is also harmonic in $\mathbf{T}_{n+1}$ for any fixed $N \in \partial \mathbf{T}_{n+1}$.

By $F_{l, n+1}$ we denote the set of continuous functions $f$ on $\partial \mathbf{T}_{n+1}=\mathbf{R}^{n}$ such that

$$
\int_{\mathbf{R}^{n}} \frac{|f(N)|}{1+|N|^{n+l-1}} d N<\infty
$$

The following Theorem 1 generalizes Theorem A, which is our result in the case $l=0$.

Theorem 1. Let $l$ be a non-negative integer and $f \in F_{l, n+1}$. Then the generalized Neumann integral $H_{l, n+1} f$ of $f$, defined in $\mathbf{T}_{n+1}$ by

$$
H_{l, n+1} f(M)=\int_{\mathbf{R}^{n}} K_{l, n+1}(M, N) f(N) d N,
$$

is a solution of the Neumann problem for $f$ and

$$
\mathcal{M}\left(\left|H_{l, n+1} f\right| ; r\right)=O\left(r^{l}\right) \quad(r \rightarrow \infty) .
$$

Remark 1 . We remark that Theorem 1 yields multiple representations in the case that $f$ satisfies (2.2) for more than one $l$. For example, if $f$ is bounded with bounded support, then (2.2) is satisfied for every non-negative integer $l$ and hence many generalized Neumann integrals $H_{l, n+1} f(l=0,1,2, \ldots)$ of $f$ are obtained. 
We shall define another Neumann kernel. The construction of our Neumann kernel is similar in spirit to Finkelstein and Scheinberg's construction for the Poisson kernel [6]. Let $\varphi(t)$ be a positive continuous function of $t \geq 1$ satisfying

$$
\varphi(1)=c_{n} / 2
$$

where $c_{n}=3(n-1) 2^{n} \alpha_{n+1}$. Denote the set

$$
\left\{t \geq 1: t^{n-1} \varphi(t)=2^{-i} c_{n}\right\}
$$

by $U_{n}(\varphi, i)(i=1,2,3, \cdots)$. Then $1 \in U_{n}(\varphi, 1)$. When there is an integer $L$ such that $U_{n}(\varphi, L) \neq \emptyset$ and $U_{n}(\varphi, L+1)=\emptyset$, we denote the set $\{i: 1 \leq i \leq L\}$ of integers by $E_{n}(\varphi)$. Otherwise, we denote the set of all positive integers by $E_{n}(\varphi)$. Let $t_{n}(i)=t_{n}(\varphi, i)$ be the minimum of elements in $U_{n}(\varphi, i)$ for each $i \in E_{n}(\varphi)$. In the former case, we put $t_{n}(L+1)=\infty$. We remark that $t_{n}(1)=1$. We define $V_{\varphi, n+1}(M, N)\left(M \in \mathbf{T}_{n+1}, N \in \partial \mathbf{T}_{n+1}\right)$ by

$$
V_{\varphi, n+1}(M, N)= \begin{cases}0 & |N|<t_{n}(1), \\ V_{i, n+1}(M, N) & t_{n}(i) \leq|N|<t_{n}(i+1) \quad\left(i \in E_{n}(\varphi)\right) .\end{cases}
$$

We put

$$
K_{\varphi, n+1}(M, N)=K_{0, n+1}(M, N)-V_{\varphi, n+1}(M, N) \quad\left(M \in \mathbf{T}_{n+1}, N \in \partial \mathbf{T}_{n+1}\right) .
$$

It is evident that $K_{\varphi, n+1}(\cdot, N)$ is also harmonic on $\mathbf{T}_{n+1}$ for any fixed $N \in \partial \mathbf{T}_{n+1}$.

To solve the Neumann problem on $\mathbf{T}_{n+1}$ for any continuous function $f$ on $\partial \mathbf{T}_{n+1}=\mathbf{R}^{n}$, we have

Theorem 2. Let $f$ be any continuous function on $\partial \mathbf{T}_{n+1}=\mathbf{R}^{n}$. Then there is a positive continuous function $\varphi(t)$ of $t \geq 1$, given explicitly in terms of the growth of $f$, such that

$$
H_{\varphi, n+1} f(M)=\int_{\mathbf{R}^{n}} K_{\varphi, n+1}(M, N) f(N) d N
$$

is a solution of the Neumann problem on $\mathbf{T}_{n+1}$ for $f$.

The following Theorem 3 extends Theorem B, which is our result in the case $l=0$.

Theorem 3. Let $k$ be a positive integer and $l$ be a non-negative integer. Let $f \in F_{l, n+1}$ and $h$ be a solution of the Neumann problem on $\mathbf{T}_{n+1}$ for $f$ such that

$$
\mathcal{M}\left(h^{+} ; r\right)=o\left(r^{k+l}\right) \quad(r \rightarrow \infty) .
$$

Then

$$
h(M)= \begin{cases}H_{l, n+1} f(M)+C & (k=1), \\ H_{l, n+1} f(M)+\Pi(X)+\sum_{j=1}^{\left[\frac{k+l}{2}\right]} \frac{(-1)^{j}}{(2 j) !} y^{2 j} \Delta^{j} \Pi(X) & (k \geq 2)\end{cases}
$$

for any $M=(X, y) \in \mathbf{T}_{n+1}$, where $C$ is a constant and $\Pi$ is a polynomial of $X=\left(x_{1}, x_{2}, \ldots, x_{n}\right) \in \mathbf{R}^{n}$ of degree less than $k+l$. 


\section{Proofs of Theorems 1, 2 and 3}

In this section we use the following notation:

$$
B_{m}(Q, r)=\left\{P \in \mathbf{R}^{m}:|P-Q|<r\right\} \quad\left(Q \in \mathbf{R}^{m}, r>0\right)
$$

and

$$
B_{m}(r)=\left\{P \in \mathbf{R}^{m}:|P|<r\right\} \quad(r>0) .
$$

First of all, we note two facts concerning $L_{k, n+1}(\rho)$. If we observe that

$$
\frac{d}{d \rho} L_{k, n+1}(\rho)=\frac{k(n+k-1)}{n} L_{k-1, n+3}(\rho) \quad(k \geq 1)
$$

from Müller [8, Lemma 13], then we have

$$
\begin{aligned}
& \frac{\partial}{\partial y}\left(c_{k, n+1}|M|^{k} L_{k, n+1}(\rho)\right)=(n-1) c_{k-1, n+2} y|M|^{k-2} L_{k, n+1}(\rho) \\
&-(n-1) c_{k-1, n+3} y|M|^{k-2} \rho L_{k-1, n+3}(\rho) \quad(k \geq 1) .
\end{aligned}
$$

We also know that

$$
\left|L_{k, m}(\rho)\right| \leq 1
$$

for any $\rho$ in (2.1), any non-negative integer $k$ and any positive integer $m \geq 2$ (see Armitage [3, Theorems C and D]).

Lemma 1. Let $l$ be a non-negative integer. For any $M \in \mathbf{T}_{n+1}$ and any $N \in \partial \mathbf{T}_{n+1}$ satisfying $2|M|<|N|$ and $|N| \geq 1$, we have

$$
\left|K_{l, n+1}(M, N)\right| \leq C_{1}(l, n)|M|^{l}|N|^{1-n-l}
$$

and

$$
\left|\frac{\partial}{\partial y} K_{l, n+1}(M, N)\right| \leq \begin{cases}C_{2}(l, n)|M|^{l-1}|N|^{1-n-l} & (l \geq 1), \\ C_{2}(0, n)|N|^{1-n} & (l=0),\end{cases}
$$

where $C_{1}(l, n)=2^{n+l-1} \alpha_{n+1}, C_{2}(l, n)=3(n-1) 2^{n+l-1} \alpha_{n+1}$ and $C_{2}(0, n)=$ $3(n-1) 2^{n} \alpha_{n+1}$.

Proof. Take any $M \in \mathbf{T}_{n+1}$ and any $N \in \partial \mathbf{T}_{n+1}$ satisfying $2|M|<|N|$ and $|N| \geq 1$. Then

$$
\begin{aligned}
\left|K_{l, n+1}(M, N)\right| & =\left.\alpha_{n+1}\left|\sum_{k=l}^{\infty} c_{k, n+1}\right| N\right|^{1-n-k}|M|^{k} L_{k, n+1}(\rho) \mid \\
& \leq \alpha_{n+1} \sum_{k=l}^{\infty} c_{k, n+1}|N|^{1-n} 2^{-k}\left(\frac{2|M|}{|N|}\right)^{k}\left|L_{k, n+1}(\rho)\right| \\
& \leq \alpha_{n+1}\left(\frac{2|M|}{|N|}\right)^{l}|N|^{1-n} \sum_{k=l}^{\infty} c_{k, n+1} 2^{-k}
\end{aligned}
$$

from (3.2). If we put $C_{1}(l, n)=2^{n+l-1} \alpha_{n+1}$, then we have (3.3). 
If $l \geq 2$, we similarly have

$$
\begin{aligned}
& \left|\frac{\partial}{\partial y} K_{l, n+1}(M, N)\right| \\
\leq & \alpha_{n+1} \sum_{k=l}^{\infty}(n-1) c_{k-1, n+2} y|M|^{k-2}|N|^{1-n-k}\left|L_{k, n+1}(\rho)\right| \\
& \quad+\alpha_{n+1} \sum_{k=l}^{\infty}(n-1) c_{k-1, n+3} y|M|^{k-2}|N|^{1-n-k}|\rho|\left|L_{k-1, n+3}(\rho)\right| \\
\leq & (n-1) \alpha_{n+1}|N|^{-n} \sum_{k=l}^{\infty} 2^{1-k}\left(\frac{2|M|}{|N|}\right)^{k-1}\left(c_{k-1, n+2}+c_{k-1, n+3}\right) \\
\leq & (n-1) \alpha_{n+1}|N|^{-n}\left(\frac{2|M|}{|N|}\right)^{l-1} \sum_{k=l}^{\infty} 2^{1-k}\left(c_{k-1, n+2}+c_{k-1, n+3}\right)
\end{aligned}
$$

from (3.1). By putting $C_{2}(l, n)=3(n-1) 2^{n+l-1} \alpha_{n+1}$, we also obtain (3.4) in the case $l \geq 2$. Since for $l=1$ or 0 ,

$$
\frac{\partial}{\partial y} K_{l, n+1}(M, N)=-\alpha_{n+1} \sum_{k=2}^{\infty} c_{k, n+1}|N|^{1-n-k} \frac{\partial}{\partial y}|M|^{k} L_{k, n+1}(\rho)
$$

we have

$$
\begin{aligned}
\left|\frac{\partial}{\partial y} K_{l, n+1}(M, N)\right| & \leq(n-1) \alpha_{n+1}|N|^{-n} \sum_{k=2}^{\infty} 2^{1-k}\left(c_{k-1, n+2}+c_{k-1, n+3}\right) \\
& \leq 3(n-1) 2^{n} \alpha_{n+1}|N|^{-n} \\
& \leq 3(n-1) 2^{n} \alpha_{n+1}|N|^{1-n} .
\end{aligned}
$$

This gives (3.4) in the case $l=1$ or 0 .

Lemma 2. Let $l$ be a non-negative integer, $\delta$ be any positive number satisfying $0<\delta<1$, and $N^{*}$ be any fixed point of $\partial \mathbf{T}_{n+1}$. Then

$$
\left|\frac{\partial}{\partial y} V_{l, n+1}(M, N)\right| \leq C\left(l, \delta, N^{*}\right) y
$$

for any $M \in B_{n+1}\left(N^{*}, \delta\right) \cap \mathbf{T}_{n+1}$ and any $N \in \partial \mathbf{T}_{n+1}$, where $C\left(l, \delta, N^{*}\right)$ is a constant depending only on $l, \delta$ and $N^{*}$.

Proof. From the definition of $V_{l, n+1}(M, N)$ and (3.1), we can evidently assume that $l \geq 3$ and $|N| \geq 1$. Then we have from (3.2) that

$$
\begin{aligned}
\left|\frac{\partial}{\partial y} V_{l, n+1}(M, N)\right| \leq & \alpha_{n+1} \sum_{k=2}^{l-1}(n-1) c_{k-1, n+2} y|M|^{k-2}|N|^{1-k-n}\left|L_{k, n+1}(\rho)\right| \\
& +\alpha_{n+1} \sum_{k=2}^{l-1}(n-1) c_{k-1, n+3} y|M|^{k-2}|N|^{1-k-n}|\rho|\left|L_{k, n+3}(\rho)\right| \\
\leq & \frac{2 y}{s_{n+1}} \sum_{k=2}^{l-1}\left(c_{k-1, n+2}+c_{k-1, n+3}\right)\left(\left|N^{*}\right|+\delta\right)^{k-2} \\
= & C\left(l, \delta, N^{*}\right) y
\end{aligned}
$$


where

$$
C\left(l, \delta, N^{*}\right)=\frac{2}{s_{n+1}} \sum_{k=2}^{l-1}\left(c_{k-1, n+2}+c_{k-1, n+3}\right)\left(\left|N^{*}\right|+\delta\right)^{k-2} .
$$

Lemma 3. Let $l$ be any non-negative integer. Let $f$ be a locally integrable function on $\partial \mathbf{T}_{n+1}$ satisfying (2.2). Then $H_{l, n+1} f$ is a harmonic function on $\mathbf{T}_{n+1}$.

Proof. For any fixed $M \in \mathbf{T}_{n+1}$, take a number $R$ satisfying $R \geq \max \{1,2|M|\}$. Then from Lemma 1 we have

$$
\int_{\mathbf{R}^{n} \backslash B_{n}(R)}\left|K_{l, n+1}(M, N)\right||f(N)| d N \leq C_{1}(l, n)|M|^{l} \int_{\mathbf{R}^{n} \backslash B_{n}(R)} \frac{|f(N)|}{|N|^{n+l-1}} d N<\infty .
$$

Thus $H_{l, n+1} f(M)$ is finite for any $M \in \mathbf{T}_{n+1}$. Since the mean value equality for $H_{l, n+1} f$ follows from Fubini's theorem, $H_{l, n+1} f(M)$ is harmonic in $\mathbf{T}_{n+1}$.

Lemma 4. Let $l$ be any non-negative integer. Let $f$ be a locally integrable and upper semicontinuous function on $\partial \mathbf{T}_{n+1}$ satisfying (2.2). Then

$$
\limsup _{M \in \mathbf{T}_{n+1}, M \rightarrow N^{*}} \frac{\partial}{\partial y} H_{l, n+1} f(M) \leq f\left(N^{*}\right)
$$

for any fixed $N^{*} \in \partial \mathbf{T}_{n+1}$.

Proof. Let $N^{*}$ be any fixed point on $\partial \mathbf{T}_{n+1}=\mathbf{R}^{n}$ and $\varepsilon$ be any positive number. Take a positive number $\delta, \delta<1$, such that

$$
f(N)<f\left(N^{*}\right)+\varepsilon
$$

for any $N \in B_{n}\left(N^{*}, \delta\right)$. From (2.2) and (3.4), we can choose a number $R^{*}, R^{*}>$ $2\left(\left|N^{*}\right|+1\right)$, such that

$$
\int_{\mathbf{R}^{n} \backslash B_{n}\left(R^{*}\right)}\left|\frac{\partial}{\partial y} K_{l, n+1}(M, N)\right||f(N)| d N<\varepsilon,
$$

for any $M \in \mathbf{T}_{n+1} \cap B_{n+1}\left(N^{*}, \delta\right)$. Put

$$
J(M)=\int_{B_{n}\left(R^{*}\right)} f(N) \frac{\partial}{\partial y} K_{0, n+1}(M, N) d N
$$

and

$$
J_{l}(M)=-\int_{B_{n}\left(R^{*}\right)} f(N) \frac{\partial}{\partial y} V_{l, n+1}(M, N) d N \quad(l \geq 0) .
$$

Since

$$
\frac{\partial}{\partial y} K_{0, n+1}(M, N)=\frac{2 y}{s_{n+1}}|M-N|^{-n-1} \quad\left(M=(X, y) \in \mathbf{T}_{n+1}, N \in \partial \mathbf{T}_{n+1}\right),
$$

we observe that

$$
\begin{aligned}
& \left|\int_{B_{n}\left(R^{*}\right) \backslash B_{n}\left(N^{*}, \delta\right)} f(N) \frac{\partial}{\partial y} K_{0, n+1}(M, N) d N\right| \\
\leq & \frac{2 y}{s_{n+1}} \int_{B_{n}\left(R^{*}\right) \backslash B_{n}\left(N^{*}, \delta\right)}|M-N|^{-n-1}|f(N)| d N \\
\leq & \frac{2 y}{s_{n+1}}\left(\frac{\delta}{2}\right)^{-n-1} \int_{B_{n}\left(R^{*}\right) \backslash B_{n}\left(N^{*}, \delta\right)}|f(N)| d N
\end{aligned}
$$


for any $M \in \mathbf{T}_{n+1} \cap B_{n+1}\left(N^{*}, \delta / 2\right)$. Since

$$
\begin{aligned}
1-\int_{B_{n}\left(N^{*}, \delta\right)} \frac{\partial}{\partial y} K_{0, n+1}(M, N) d N & =\int_{\mathbf{R}^{n} \backslash B_{n}\left(N^{*}, \delta\right)} \frac{\partial}{\partial y} K_{0, n+1}(M, N) d N \\
& =\frac{2 y}{s_{n+1}} \int_{\mathbf{R}^{n} \backslash B_{n}\left(N^{*}, \delta\right)}|M-N|^{-n-1} d N
\end{aligned}
$$

for any $M \in \mathbf{T}_{n+1}$ (see Armitage and Gardiner [4, p. 24]), we have

$$
\lim _{M \rightarrow N^{*}, M \in \mathbf{T}_{n+1}} \int_{B_{n}\left(N^{*}, \delta\right)} \frac{\partial}{\partial y} K_{0, n+1}(M, N) d N=1 .
$$

Finally (3.5), (3.7) and (3.8) yield

$$
\limsup _{M \rightarrow N^{*}, M \in \mathbf{T}_{n+1}} J(M) \leq f\left(N^{*}\right)+\varepsilon .
$$

From Lemma 2 we obtain

$$
\begin{aligned}
\left|J_{l}(M)\right| & \leq \int_{B_{n}\left(R^{*}\right)}|f(N)|\left|\frac{\partial}{\partial y} V_{l, n+1}(M, N)\right| d N \\
& \leq \int_{B_{n}\left(R^{*}\right)} C\left(l, \delta, N^{*}\right) y|f(N)| d N \\
& \leq C_{3} y
\end{aligned}
$$

for any $M \in \mathbf{T}_{n+1} \cap B_{n+1}\left(N^{*}, \delta\right)$, where

$$
C_{3}=C\left(l, \delta, N^{*}\right) \int_{B_{n}\left(R^{*}\right)}|f(N)| d N .
$$

These and (3.6) yield

$$
\begin{aligned}
& \limsup _{M \rightarrow N^{*}, M \in \mathbf{T}_{n+1}} \frac{\partial}{\partial y} H_{l, n+1} f(M) \\
= & \limsup _{M \rightarrow N^{*}, M \in \mathbf{T}_{n+1}} \int_{\mathbf{R}^{n}} f(N) \frac{\partial}{\partial y} K_{l, n+1}(M, N) d N \\
= & \limsup _{M \rightarrow N^{*}, M \in \mathbf{T}_{n+1}}\left(J(M)+J_{l}(M)+\int_{\mathbf{R}^{n} \backslash B_{n}\left(R^{*}\right)} f(N) \frac{\partial}{\partial y} K_{l, n+1}(M, N) d N\right) \\
\leq & f\left(N^{*}\right)+2 \varepsilon .
\end{aligned}
$$

Now the conclusion immediately follows.

Proof of Theorem 1. It immediately follows from Lemmas 3 and 4 that $H_{l, n+1} f$ is a harmonic function on $\mathbf{T}_{n+1}$ and

$$
\lim _{M \rightarrow N^{*}, M \in \mathbf{T}_{n+1}} \frac{\partial}{\partial y} H_{l, n+1} f(M)=f\left(N^{*}\right)
$$

for any $N^{*} \in \partial \mathbf{T}_{n+1}$. 
We now turn to the proof of (2.3). For any positive number $r>1$ we have

$$
\begin{aligned}
\frac{s_{n+1} r^{n}}{2} \mathcal{M}\left(\left|H_{l, n+1} f\right| ; r\right) & =\int_{\sigma_{n+1}(r)}\left|\int_{\mathbf{R}^{n}} K_{l, n+1}(M, N) f(N) d N\right| d \sigma_{M} \\
& \leq \int_{\sigma_{n+1}(r)} \int_{\mathbf{R}^{n}}\left|K_{l, n+1}(M, N) f(N)\right| d N d \sigma_{M} \\
& =\int_{\mathbf{R}^{n}} \int_{\sigma_{n+1}(r)}\left|K_{l, n+1}(M, N) f(N)\right| d \sigma_{M} d N \\
& =T_{1, l}(r)+T_{2, l}(r),
\end{aligned}
$$

where

$$
T_{1, l}(r)=\int_{\mathbf{R}^{n} \backslash B_{n}(2 r)} \int_{\sigma_{n+1}(r)}\left|K_{l, n+1}(M, N) f(N)\right| d \sigma_{M} d N
$$

and

$$
T_{2, l}(r)=\int_{B_{n}(2 r)} \int_{\sigma_{n+1}(r)}\left|K_{l, n+1}(M, N) f(N)\right| d \sigma_{M} d N .
$$

We note that if $l \geq 1$ and $1 \leq|N|<2|M|$, then

$$
\begin{aligned}
\left|V_{l, n+1}(M, N)\right| & \leq \alpha_{n+1} \sum_{k=0}^{l-1} c_{k, n+1}|N|^{1-k-n}|M|^{k}\left|L_{k, n+1}(\rho)\right| \\
& \leq \alpha_{n+1}|N|^{1-n} \sum_{k=0}^{l-1} 2^{-k} c_{k, n+1}\left(\frac{2|M|}{|N|}\right)^{k} \\
& \leq C_{4}|N|^{2-l-n}|M|^{l-1}
\end{aligned}
$$

where

$$
C_{4}=2^{l-1} \alpha_{n+1} l \max _{0 \leq k \leq l-1} 2^{-k} c_{k, n+1} .
$$

Hence we have

$$
\begin{gathered}
\int_{B_{n}(2 r)}|f(N)| \int_{\sigma_{n+1}(r)}\left|V_{l, n+1}(M, N)\right| d \sigma_{M} d N \\
\leq 2^{-1} C_{4} s_{n+1} r^{n+l-1} \int_{B_{n}(2 r) \backslash B_{n}(1)} \frac{|f(N)|}{|N|^{n+l-2}} d N=C_{5} r^{n+l},
\end{gathered}
$$

where

$$
C_{5}=C_{4} s_{n+1} \int_{\mathbf{R}^{n} \backslash B_{n}(1)} \frac{|f(N)|}{|N|^{n+l-1}} d N \quad(<\infty) .
$$

Since

$$
\frac{1}{s_{n+1} r^{n}} \int_{S_{n+1}(r)}|M-N|^{1-n} d \sigma_{M} \leq r^{1-n}
$$

(see Armitage and Gardiner [4, p. 99]), we obtain

$$
\begin{gathered}
\int_{B_{n}(2 r)}|f(N)| \int_{\sigma_{n+1}(r)}\left|K_{0, n+1}(M, N)\right| d \sigma_{M} d N \leq 2^{-1} \alpha_{n+1} s_{n+1} r \int_{B_{n}(2 r)}|f(N)| d N \\
\leq 2^{-1}(n-1)^{-1} r \int_{B_{n}(2 r)} \frac{2(2 r)^{n+l-1}}{1+|N|^{n+l-1}}|f(N)| d N \leq C_{6} r^{n+l},
\end{gathered}
$$

where

$$
C_{6}=2^{n+l-1}(n-1)^{-1} \int_{\mathbf{R}^{n}} \frac{|f(N)|}{1+|N|^{n+l-1}} d N .
$$


These immediately yield

$$
\begin{aligned}
T_{2, l}(r) & \leq \int_{B_{n}(2 r)}|f(N)| \int_{\sigma_{n+1}(r)}\left(\left|K_{0, n+1}(M, N)\right|+\left|V_{l, n+1}(M, N)\right|\right) d \sigma_{M} d N \\
& \leq\left(C_{5}+C_{6}\right) r^{n+l} .
\end{aligned}
$$

From Lemma 1 we easily see that

$$
T_{1, l}(r) \leq 2^{-1} C_{1}(l, n) s_{n+1} r^{n+l} \int_{\mathbf{R}^{n} \backslash B_{n}(2 r)} \frac{|f(N)|}{|N|^{n+l-1}} d N \leq C_{7} r^{n+l},
$$

where

$$
C_{7}=2^{-1} C_{1}(l, n) s_{n+1} \int_{\mathbf{R}^{n} \backslash B_{n}(1)} \frac{|f(N)|}{|N|^{n+l-1}} d N .
$$

These give (2.3).

To prove Theorem 2, we need

Lemma 5. Let $\varphi(t)$ be a positive continuous function of $t \geq 1$ satisfying $\varphi(1)=$ $c_{n} / 2$. Then for any $M \in \mathbf{T}_{n+1}$ and any $N \in \partial \mathbf{T}_{n+1}$ satisfying $|N|>\max \{1,4|M|\}$,

$$
\left|K_{\varphi, n+1}(M, N)\right|<\varphi(|N|)
$$

and

$$
\left|\frac{\partial}{\partial y} K_{\varphi, n+1}(M, N)\right|<4 \varphi(|N|) .
$$

Proof. Take any $M \in \mathbf{T}_{n+1}$ and any $N \in \partial \mathbf{T}_{n+1}$ satisfying $|N|>\max \{1,4|M|\}$. Choose an integer $i_{0} \in E_{n}(\varphi)$ such that $t_{n}\left(i_{0}\right) \leq|N|<t_{n}\left(i_{0}+1\right)$. Then

$$
K_{\varphi, n+1}(M, N)=K_{i_{0}, n+1}(M, N) .
$$

From Lemma 1 we easily see that

$$
\left|K_{i_{0}, n+1}(M, N)\right| \leq C_{1}\left(i_{0}, n\right)|M|^{i_{0}}|N|^{1-n-i_{0}} \leq C_{1}\left(i_{0}, n\right) 2^{-2 i_{0}}|N|^{1-n} .
$$

Hence

$$
\left|K_{\varphi, n+1}(M, N)\right| \leq C_{1}\left(i_{0}, n\right) 2^{-2 i_{0}}|N|^{1-n} \leq \varphi(|N|) .
$$

In the same way we can also see (3.11) by applying Lemma 1 to $\frac{\partial}{\partial y} K_{i_{0}, n+1}(M, N)$.

Proof of Theorem 2. Let $(t, \Theta)$ be the spherical coordinates in $\mathbf{R}^{n}$. We identify $(1, \Theta) \in \mathbf{S}^{n-1}$ with $\Theta$. Put

$$
C_{8}=\frac{c_{n}}{2} \max \left\{1, \int_{\mathbf{S}^{n-1}}|f(1, \Theta)| d \Theta\right\}
$$

and

$$
\psi(t)= \begin{cases}C_{8} t^{-n-1}\left(\int_{\mathbf{S}^{n-1}}|f(t, \Theta)| d \Theta\right)^{-1} & \left(\int_{\mathbf{S}^{n-1}}|f(t, \Theta)| d \Theta>0\right), \\ \infty & \left(\int_{\mathbf{S}^{n-1}}|f(t, \Theta)| d \Theta=0\right)\end{cases}
$$

for $t \geq 1$, where $d \Theta$ is the surface element of $\mathbf{S}^{n-1}$ at $(1, \Theta) \in \mathbf{S}^{n-1}$. If we define $\varphi(t)(t \geq 1)$ by

$$
\varphi(t)=\min \left\{\frac{c_{n}}{2}, \psi(t)\right\}
$$


then $\varphi(t)$ is a positive continuous function satisfying $\varphi(1)=c_{n} / 2$. For any fixed $M \in \mathbf{T}_{n+1}$ we can choose a number $R_{1}>\max \{1,4|M|\}$ such that

$$
\begin{gathered}
\int_{\mathbf{R}^{n} \backslash B_{n}\left(R_{1}\right)}\left|K_{\varphi, n+1}(M, N) f(N)\right| d N \\
\leq \int_{R_{1}}^{\infty}\left(\int_{\mathbf{S}^{n-1}}|f(t, \Theta)| d \Theta\right) \varphi(t) t^{n-1} d t \leq C_{8} \int_{R_{1}}^{\infty} t^{-2} d t<\infty
\end{gathered}
$$

from Lemma 5. It is evident that

$$
\int_{B_{n}\left(R_{1}\right)}\left|K_{\varphi, n+1}(M, N) f(N)\right| d N<\infty .
$$

These give that

$$
\int_{\mathbf{R}^{n}}\left|K_{\varphi, n+1}(M, N) f(N)\right| d N<\infty .
$$

To see that $H_{\varphi, n+1} f(M)$ is harmonic in $\mathbf{T}_{n+1}$, we observe from Fubini's theorem that $H_{\varphi, n+1} f(M)$ has the locally mean-value property.

Finally we shall show that

$$
\lim _{M \in \mathbf{T}_{n+1}, M \rightarrow N^{*}} \frac{\partial}{\partial y} H_{\varphi, n+1} f(M)=f\left(N^{*}\right)
$$

for any fixed $N^{*} \in \partial \mathbf{T}_{n+1}$. In a similar way to (3.12) we also have

$$
\int_{\mathbf{R}^{n} \backslash B_{n}\left(R_{1}\right)}\left|\frac{\partial}{\partial y} K_{\varphi, n+1}(M, N) f(N)\right| d N<\infty
$$

for any fixed $M \in \mathbf{T}_{n+1}$ and any number $R_{1}>\max \{1,4|M|\}$. Let $\varepsilon$ be any positive number. Choose a sufficiently large number $R^{*}\left(R^{*}>4\left(\left|N^{*}\right|+1\right)\right)$ such that

$$
\int_{\mathbf{R}^{n} \backslash B_{n}\left(R^{*}\right)}\left|\frac{\partial}{\partial y} K_{\varphi, n+1}(M, N) f(N)\right| d N<\varepsilon .
$$

Since $f$ is continuous on $\partial \mathbf{T}_{n+1}$, take a positive number $\delta(\delta<1)$ such that

$$
f(N)<f\left(N^{*}\right)+\varepsilon
$$

for any $N \in B_{n}\left(N^{*}, \delta\right)$. In the completely same way as the proof of Lemma 4, we also obtain

$$
\limsup _{M \in \mathbf{T}_{n+1}, M \rightarrow N^{*}} \int_{B_{n}\left(R^{*}\right)} f(N) \frac{\partial}{\partial y} K_{0, n+1}(M, N) d N \leq f\left(N^{*}\right)+\varepsilon .
$$

If we take an integer $i_{0} \in E_{n}(\varphi)$ satisfying $t_{n}\left(i_{0}\right) \leq R^{*}<t_{n}\left(i_{0}+1\right)$, then we see from Lemma 2 that

$$
\begin{gathered}
\int_{B_{n}\left(R^{*}\right)}|f(N)|\left|\frac{\partial}{\partial y} V_{\varphi, n+1}(M, N)\right| d N \leq \int_{B_{n}\left(R^{*}\right)} \sum_{i=1}^{i_{0}}\left|\frac{\partial}{\partial y} V_{i, n+1}(M, N) f(N)\right| d N \\
\leq y \int_{B_{n}\left(R^{*}\right)} \sum_{i=1}^{i_{0}} C\left(i, \delta, N^{*}\right)|f(N)| d N=C_{9} y
\end{gathered}
$$

for any $M \in B_{n+1}\left(N^{*}, \delta\right) \cap \mathbf{T}_{n+1}$, where $C_{9}$ is a constant. These yield

$$
\limsup _{M \in \mathbf{T}_{n+1}, M \rightarrow N^{*}} \int_{\mathbf{R}^{n}} f(N) \frac{\partial}{\partial y} K_{\varphi, n+1}(M, N) d N \leq f\left(N^{*}\right)+2 \varepsilon .
$$


By replacing $f$ with $-f$, we also have

$$
\liminf _{M \in \mathbf{T}_{n+1}, M \rightarrow N^{*}} \int_{\mathbf{R}^{n}} f(N) \frac{\partial}{\partial y} K_{\varphi, n+1}(M, N) d N \geq f\left(N^{*}\right)-2 \varepsilon .
$$

From these, (3.13) follows immediately.

To prove Theorem 3 completely we shall first give an easy proof of the following lemma, which is proved in a different way from Armitage [1].

Lemma 6 (Armitage [1, Lemma 2]). If $H(M)$ is a harmonic polynomial of $M=$ $(X, y) \in \mathbf{R}^{n+1}$ of degree $m$ and $\partial H / \partial y$ vanishes on $\partial \mathbf{T}_{n+1}$, then there is a polynomial $\Pi$ of $X \in \mathbf{R}^{n}$ of degree $m$ such that

$$
H(X, y)= \begin{cases}\Pi(X)+\sum_{j=1}^{\left[\frac{1}{2} m\right]} \frac{(-1)^{j}}{(2 j) !} y^{2 j} \Delta^{j} \Pi(X) & (m \geq 2), \\ \Pi(X) & (m=0,1)\end{cases}
$$

Proof. Put

$$
H(X, y)=\Pi_{0}(X)+\Pi_{1}(X) y+\cdots+\Pi_{m}(X) y^{m} \quad\left((X, y) \in \mathbf{R}^{n+1}\right),
$$

where $\Pi_{j}(X)$ is a polynomial of $X \in \mathbf{R}^{n}$ of degree at most $m-j$. We remark that a sequence of the equations

$$
\Pi_{j}(X)=-j^{-1}(j-1)^{-1} \Delta \Pi_{j-2}(X) \quad(j=2,3, \cdots, m)
$$

and

$$
\Pi_{1}(X)=0
$$

follows from

$$
\Delta H=0 \quad \text { on } \mathbf{R}^{n+1} \quad \text { and } \quad \partial H / \partial y=0 \quad \text { on } \mathbf{R}^{n},
$$

respectively. If we set $\Pi(X)=\Pi_{0}(X)$ on $\mathbf{R}^{n}$, then

$$
H(X, y)=\Pi(X)+\sum_{j=1}^{\left[\frac{1}{2} m\right]} \frac{(-1)^{j}}{(2 j) !} y^{2 j} \Delta^{j} \Pi(X)
$$

from (3.14), (3.15) and (3.16).

Proof of Theorem 3. Suppose that $f$ and $h$ are two functions given in Theorem 3 . Then we know from Theorem 1 that $h-H_{l, n+1} f$ has a harmonic continuation $H$ to $\mathbf{R}^{n+1}$ such that $H$ is an even function of $y$ (see Armitage [2, 88.2$]$ ). Now we have

$$
\begin{aligned}
\mathcal{M}\left(H^{+} ; r\right) & =\mathcal{M}\left(\left(h-H_{l, n+1} f\right)^{+} ; r\right) \\
& \leq \mathcal{M}\left(h^{+} ; r\right)+\mathcal{M}\left(\left|H_{l, n+1} f\right| ; r\right) \\
& =o\left(r^{k+l}\right)+o\left(r^{l+1}\right)=o\left(r^{k+l}\right) \quad(r \rightarrow \infty),
\end{aligned}
$$

by (2.4) and (2.3) of Theorem 1. This implies that $H$ is a polynomial of degree less than $k+l$ (see Brelot [5, Appendix]). The conclusions of the theorem follow immediately from Lemma 6 . 


\section{REFERENCES}

1. D.H. Armitage, The Neumann problem for a function harmonic in $\mathbf{R}^{n} \times(0, \infty)$, Arch. Rational Mech. Anal., 63 (1976), 89-105. MR0427656 (55:687)

2. __ On harmonic polynomials, Proc. London Math. Soc., (3) 34 (1977), 173-192. MR 0427661 (55:692)

3. 38 (1979), 53-71. MR520972 (80d:31003)

4. D.H. Armitage and S.J. Gardiner, Classical Potential Theory, Springer-Verlag London Ltd., 2001. MR 1801253 (2001m:31001)

5. M. Brelot, Éléments de la théorie classique du potentiel, Centre de Documentation Universitaire, Paris, 1965. MR0178154 (31:2412)

6. M. Finkelstein and S. Scheinberg, Kernels for solving problems of Dirichlet type in a halfplane, Advances in Math., 18 (1975), 108-113. MR0382677(52:3559)

7. S.J. Gardiner, The Dirichlet and Neumann problems for harmonic functions in half-spaces, J. London Math. Soc., (2) 24 (1981), 502-512. MR635881 (82m:31006)

8. C. Müller, Spherical harmonics, Springer-Verlag, Berlin, 1966. MR0199449 (33:7593)

9. D. Siegel and E. Talvila, Sharp growth estimates for modified Poisson integrals in a half space, Potential Anal., 15 (2001), 333-360. MR1856153(2002h:31008)

10. H. Yoshida, A type of uniqueness for the Dirichlet problem on a half-space with continuous data, Pacific J. Math., 172 (1996), 591-609. MR.1386632 (97g:31007)

Rakuten, Inc., 604, 1778-1 Hisasue, Takatsu-Ku, Kawasaki-Shi, Kanagawa 213-0026, JAPAN

E-mail address: fumiyama.shu@mail.rakuten.com.tw

Department of Mathematics and Informatics, Division of Fundamental Science, Graduate School of Science, Chiba University, 1-33 Yayoi-cho, Inage-Ku, Chiba 263-8522, JAPAN

E-mail address: emblem-yp5@graduate.chiba-u.jp

Department of Mathematics and Informatics, Division of Fundamental Science, Graduate School of Science, Chiba University, 1-33 Yayoi-cho, Inage-ku, Chiba 263-8522, JAPAN

E-mail address: myanagis@g.math.s.chiba-u.ac.jp 